\title{
The CODALEMA/EXTASIS experiment: a multi-scale and multi-wavelength instrument for radio-detection of extensive air-showers
}

\author{
B. Revenu ${ }^{1,3}$, D. Charrier ${ }^{1,3}$, R. Dallier ${ }^{1,3}$, A. Escudie ${ }^{1}$, D.García-Fernández ${ }^{1}$, A. \\ Lecacheux $^{2}$, and L. Martin ${ }^{1,3}$ \\ ${ }^{1}$ Subatech, Institut Mines-Telecom Atlantique, CNRS, Universite de Nantes, Nantes, France \\ ${ }^{2}$ CNRS-Observatoire de Paris, Meudon, France \\ ${ }^{3}$ Unité Scientifique de Nançay, Observatoire de Paris, CNRS, PSL, UO/OSUC, Nançay, France \\ E-mail: revenuain2p3.fr
}

Hosted by the Nançay Radio Observatory, the CODALEMA experiment is dedicated to radio detection of cosmic ray induced extensive air showers. It is composed of:

- 57 self-triggering radio detection stations working in the $20-200 \mathrm{MHz}$ band and spread over $1 \mathrm{~km}^{2}$;

- an array of 13 scintillators as particle detector;

- a compact array made of 10 cabled antennas, triggered by the particle detector;

- a small array of 5 cabled antennas, and whose role is to figure out the capabilities of a phased antenna cluster to cleverly select air shower events;

- a 3D detector, measuring the electric field in three orthogonal polarizations.

Also supported by CODALEMA is the R\&D EXTASIS project, aiming at detecting the lowfrequency signal (below $9 \mathrm{MHz}$ ) produced at the sudden disappearance of the air shower particles hitting the ground. All these antenna arrays present different density and extent, and can be operated in a joint mode to record simultaneously the radio signal coming from an air shower. Therefore, the Nançay facilities may offer a complete description of the air shower induced electric field at small, medium and large scale, and over an unique and very wide frequency band (from 2 to $200 \mathrm{MHz}$ ). We describe the current instrumental set-up and the performances of CODALEMA/EXTASIS. 


\section{Radio signal and cosmic rays}

Cosmic rays entering our atmosphere interact with the air molecules and produce atmospheric air showers. These showers are constituted by many secondary particles, in particular electrons and positrons radiating a detectable electric field. The emission mechanisms are now well understood $[1,2]$ and regularly observed in various experiments $[3,4,5] \ldots$ This radio signal can be compared to theoretical expectations through simulations [6, 7], in order to successfully compute the energy of the primary cosmic ray and its nature. The goal is to provide an accurate estimation of the cosmic ray composition above $10^{16} \mathrm{eV}$. This composition is still poorly known but we expect large progress in the next decade due to the use of the radio signal together with improved cosmic ray observatories such as the Pierre Auger Observatory [8].

It has been shown that the radio signal allows to estimate the nature of the primary cosmic ray through the measurement of the atmospheric depth of the maximum of the shower development $X_{\max }[9,10]$. In LOFAR for instance, which has a high density of antennas spread over a small area $\left(0.2 \mathrm{~km}^{2}\right)$, the uncertainty on $X_{\max }$ is $17 \mathrm{~g} / \mathrm{cm}^{2}$ [11], similar to what is done using with the fluorescence technique ( $\sim 20 \mathrm{~g} / \mathrm{cm}^{2}$ [12]) but with a duty cycle close to $100 \%$ compared to the $14 \%$ [13] of a fluorescence telescope.

The detection of this very rich radio signal is realized in various frequency bands: $20-$ $200 \mathrm{MHz}$ for CODALEMA, $30-80 \mathrm{MHz}$ for AERA and Tunka-Rex, $30-80$ or (exclusive) $110-190 \mathrm{MHz}$ for LOFAR. This is not an easy task as the current experiments rely either on external triggering thanks to a joint shower detector (particles or fluorescence) sensitive only to air showers or on internal triggering with the strong drawback that a huge fraction of the data is generated by noise (of anthropic or natural origin). In Nançay, we aim at efficiently detecting air showers with a very large frequency band in order to fully characterize the primary cosmic ray independently of any other shower detector. This will allow us to contribute to the cosmic ray domain (spectrum and composition) in the domain $10^{16}-10^{18} \mathrm{eV}$.

\section{The CODALEMA/EXTASIS experiment at Nançay}

The study of air showers created by high energy cosmic rays interacting in our atmosphere is ongoing since 15 years in Nançay. The instruments were upgraded several times and today, we run a total of 5 arrays of various detectors. Only one of them is using the well-established surface detector technique using scintillators, already in use at the time of the second period of the CODALEMA experiment. The others are all observing the radio counterpart of the air shower phenomenon. In FIG. 1, we display the ground map of the current setups, together with the location of the FR606 LOFAR station and the NenuFAR (New Extension in Nançay Upgrading LOFAR) radiotelescope, as a SKA pathfinder.

\section{The scintillator detector array (SD)}

The SD provides the classical reconstruction of air showers: energy, arrival direction and core position. These informations are used to help/confirm the shower detection and reconstruction using the radio signal. The 13 scintillators are spread over an area of $340 \times 340 \mathrm{~m}^{2}$; its energy 


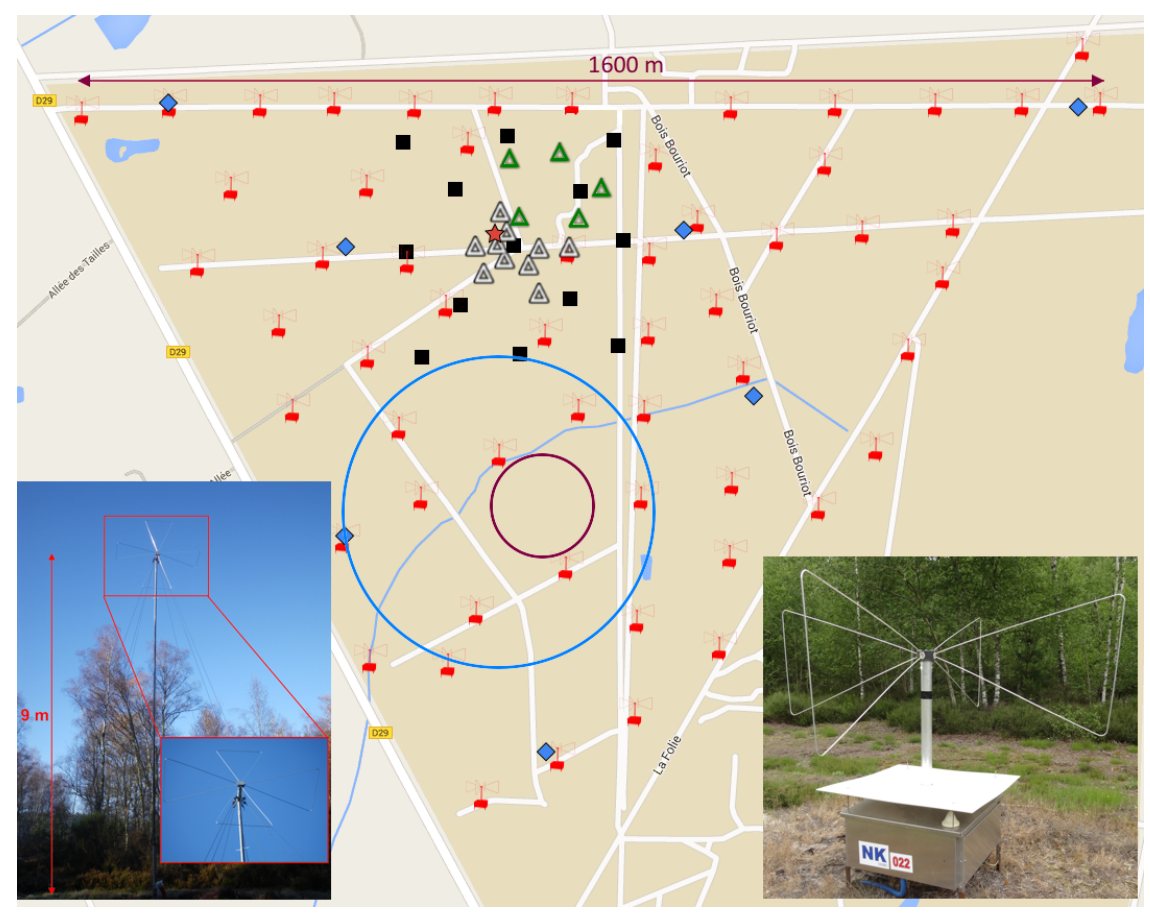

Figure 1: The CODALEMA/EXTASIS instruments cover an area of $1 \mathrm{~km}^{2}$. Black squares are the scintillator positions of the particle array. The small red radio station symbols corresponds to the 57 autonomous stations (self-triggered). White triangles stand for the compact array (externally-triggered); green triangles are the antennas of the continuous array (self-triggered). The blue squares are the low frequency antennas of the EXTASIS experiment (externally triggered). The red star indicates the tripole antenna (externallytriggered). The picture of the bottom left is one of the EXTASIS detector. The picture of the bottom right is one of the 57 autonomous radio stations.

threshold is roughly around the knee $\left(10^{15} \mathrm{eV}\right)$ and is fully efficient above $10^{16} \mathrm{eV}$. It has a very low background as scintillators are sensitive to high energy particles of the shower only. The DAQ of the scintillators is able to trigger in real time other instruments throughout the observatory: a time-tagging station holding a GPS antenna for accurate datation, the compact radio array (see section 5), the tripole antenna (see section 6), the EXTASIS detectors (see section 7).

\section{The autonomous stations radio array}

The largest instrument dedicated to cosmic-ray detection in Nançay is the array of 57 autonomous stations, covering the full area of the observatory $\left(1 \mathrm{~km}^{2}\right)$. The typical distance between two neighbouring stations is $150 \mathrm{~m}$. These stations sample the incoming electric field in both horizontal east-west (EW) and north-south (NS) polarizations. We use the butterfly antenna [14], also in use in AERA. Both input channels pass into a splitter. One part is filtered in the $35-80$ and $150-200 \mathrm{MHz}$ bands and is compared to a voltage threshold. The other is continuously digitized at a sampling rate of $1 \mathrm{GHz}$. If the filtered analogic signal is larger than the threshold, this constitutes the level 1 trigger T1: we record the GPS time of the event together will the full-band signal in both channels, during $2.56 \mu \mathrm{s}$. We can choose the T1 conditions independently on each polarization (OR or AND). At the level of the trigger board, the maximum trigger rate is of the order 
of $10^{3}$ events per second. When selected by a second-level trigger T2, digitized data are read; this part of the process increases significantly the dead time and the maximum trigger rate decreases to roughly 28 events per second. The selection can be done at the station level by applying a cut on the rise time of the signal as the transients produced by air showers are known to last 10-20 ns only. A third-level trigger (T3) is currently in use in Nançay since more than a year: it is a selection on several stations having T2s with timestamps in agreement with the propagation of waves at the speed of light as it is the case for air shower radio signals. The resulting average T3 rate is around $1 \mathrm{~Hz}$ with some peaks at $20 \mathrm{~Hz}$. The average number of radio transients corresponding to actual shower is 2 per day. They are identified through the time coincidence with the SD and also with the polarization pattern of the hit antennas. The energy threshold for the radio-detected cosmic rays is around $10^{17} \mathrm{eV}$. These autonomous stations are unique among those in use in other radio experiments on cosmic ray. The frequency band is very large $20-80 \mathrm{MHz}$ and $110-200 \mathrm{MHz}$ at once, associated with a fast sampling rate of $1 \mathrm{GHz}$. Measuring the electric field up to $200 \mathrm{MHz}$ is important as the Cherenkov contribution clearly appears in the high frequency domain (above $100 \mathrm{MHz})$. Some results are presented in [3].

\section{The Compact Radio Array}

The compact array allows to study the small-scale variations of the electric field. We are using two separated arrays.

\subsection{Externally triggered compact array}

We are running a cluster of 10 antennas spread over an area of $150 \times 150 \mathrm{~m}^{2}$. These antennas detect the electric field in two linear horizontal polarizations. They are triggered by the SD; 20 channels are recorded over $6 \mu \mathrm{s}$, with a sampling rate of $400 \mathrm{MHz}$ and a in the frequency band 10-200 MHz. Some results of this array are presented in [15].

\subsection{Self triggered compact array}

We also use a cluster of 5 antennas working in a self-trigger mode. Its main characteristics is to sample the sky in a continuous way: there is no trigger. This sampling is done on 8 channels in circular polarization after superposition of two linear polarizations added in quadrature. The sampling rate is $100 \mathrm{MHz}$ with a bandwidth of $50 \mathrm{MHz}$, between 30 and $80 \mathrm{MHz}$. Data are stored in a ring buffer and analyzed in real time with a GPU (NVIDIA K20 at 5 Tflops). The data are first filtered, we merge the data into a circular polarized channel and the signal envelope is computed for each time window of $500 \mathrm{~ns}$, corresponding to the time aperture of the array, We generate 2000 beams on the sky (by shifting the individual signals) with beamforming. Then we search for beams having an amplitude above a defined threshold. The beam forming technique increases the sensitivity by a factor $\sqrt{N}, N$ being the number of channels in use. A strong advantage of this continuous sampling is that the dead time is null. Work is in progress.

\section{3D electric field measurements}

We measure at one location in Nançay the incoming electric field in coincidence with the SD. This detector is close to the center of the SD and contains three antennas allowing the electric field 
measurement in the three EW, NS and vertical polarizations. The goals of this 3D measurement are:

- to get the full electric field directly (i.e. avoid to estimate the vertical component from the measurement of two horizontal polarizations as it is done in most experiments);

- to determine wether or not the electric field emitted by air showers can be described in the far-field hypothesis.

The design of the 3D detector is based on three butterfly antennas with their LONAMOS LNA (Low Noise Amplifier) rotated twice around the NS and vertical axis. It is triggered by the SD and the three channels are recorded with a sampling rate of $100 \mathrm{MHz}$ during $2.56 \mu \mathrm{s}$. A picture of the 3D detector is presented in FIG. 2. The results of this detector are presented in [16].

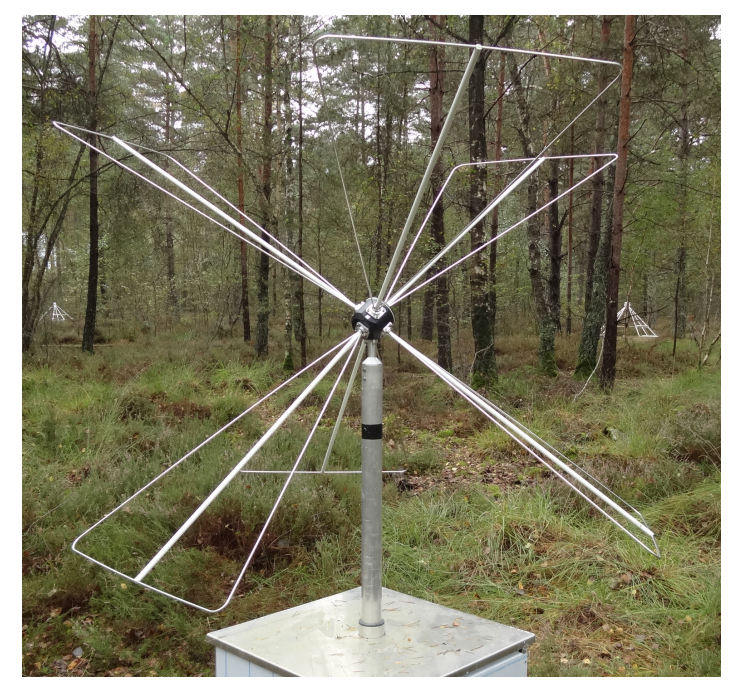

Figure 2: Picture of the 3D detector, whose location is indicated by the red star in FIG. 1.

\section{EXTASIS: search for the low frequency radio signal}

The most recent radio detector installed in Nançay is the EXTASIS experiment. EXTASIS stands for EXTinction of Air Shower Induced Signal and its main goal is to detect the electric field emitted by air showers when their front hits the ground. Secondary electrons and positrons are very quickly stopped after a few $\mathrm{cm}$ when reaching the ground. This phenomenon generates a large electric field as the time and space derivatives of the charge density and current in the shower are large. This "sudden death" signal is expected to peak at low frequency (1-10 MHz), as discussed in $[17,18]$. Apart from this very specific signal, a low frequency detector should also detect the "regular" electric field emitted during the shower development while still in the air. The theoretical potential of the electric field from the sudden death of shower is huge: detection at large distances (i.e. possibility to have a sparse and cheap array), core position determination very easy and precise, direct $X_{\max }$ measurement. In reality, things are always more complex: the frequency band 1-10 MHz is overcrowded; many man-made emitters use this band and the atmospheric noise 
background is also very large, in particular during the night because of the ionosphere's daily variations. The current low frequency setup of EXTASIS consists today in 7 butterfly antennas installed at $9 \mathrm{~m}$ above the ground (as indicated by simulations of the antenna response at these frequencies), in both EW and vertical polarizations. These antennas are triggered by the SD and their data are digitized by oscilloscopes with a sampling rate of $500 \mathrm{MHz}$ during 2 ms over 8 bits. The LNA associated to the antennas is a modified version of the LONAMOS LNA. One of the low frequency detector can be seen in FIG. 3. The first results are presented in [19].

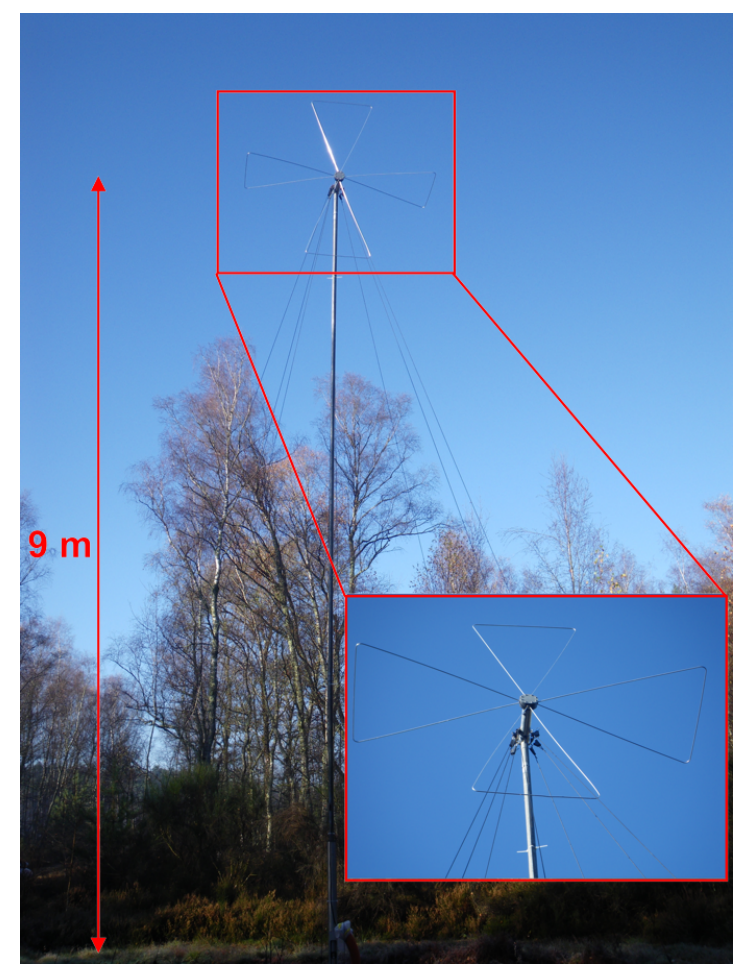

Figure 3: Picture of one of the low frequency detector of EXTASIS.

\section{Conclusion}

The CODALEMA/EXTASIS experiment in Nançay allows to detect air showers initiated by high-energy cosmic rays in a very wide frequency band: 1-200 MHz. This permits to have the full picture of the radio emission of showers and the various emission mechanisms in play: the geomagnetic effect, the charge-excess effect and the sudden death signal. This will allow a better reconstruction of the primary cosmic rays characteristics, in particular its nature. The first lowfrequency events associated with cosmic rays are detected since a year and some results are shown in [19].

\section{Acknowledgements}

We thank the Région Pays de la Loire for its financial support of the Astroparticle group of Subatech and in particular for its contribution to the EXTASIS experiment. 


\section{References}

[1] D. Ardouin et al. Geomagnetic origin of the radio emission from cosmic ray induced air showers observed by CODALEMA. Astropart.Phys., 31(3):192-200, 2009.

[2] The Pierre Auger Collaboration. Probing the radio emission from cosmic-ray-induced air showers by polarization measurements. Phys. Rev. D, 89(052002), March 2014.

[3] L. Martin et al. Main features of cosmic ray induced air showers measured by the CODALEMA experiment. In this conference.

[4] C. Glaser et al. Results and Perspectives of the Auger Engineering Radio Array. In Proceedings of the ARENA 2016 workshop (Groningen, The Netherlands). ARENA, 2016.

[5] P. A. Bezyazeekov et al. Radio measurements of the energy and the depth of the shower maximum of cosmic-ray air showers by Tunka-Rex. JCAP, 1601(01):052, 2016.

[6] Vincent Marin et al. Simulation of radio emission from cosmic ray air shower with SELFAS2. Astropart.Phys., 35:733-741, 2012.

[7] T. Huege et al. Full Monte Carlo simulations of radio emission from extensive air showers with CoREAS. In proceedings of the 33rd ICRC, Rio de Janeiro, Brasil, number id 548. arXiv:1307.7566, July 2013.

[8] R. Engel. Upgrade of the Pierre Auger Observatory. In ICRC, 2015.

[9] F. Gaté et al. $X_{\text {max }}$ reconstruction from amplitude information with AERA. In Proceedings of the ARENA 2016 workshop (Groningen, The Netherlands). ARENA, 2016.

[10] S. Buitink et al. A large light-mass component of cosmic rays at $10^{17}-10^{17.5}$ electronvolts from radio observations. Nature, 531:70-73, March 2016.

[11] S. Buitink et al. A large light-mass component of cosmic rays at $10^{17}--10^{17.5}$ electronvolts from radio observations. Nature, 531:70, 2016.

[12] J. Abraham et al. Measurement of the Depth of Maximum of Extensive Air Showers above $10^{18} \mathrm{eV}$. Phys.Rev.Lett., 104:091101, 2010.

[13] J. Abraham et al. The Fluorescence Detector of the Pierre Auger Observatory. Nucl.Instrum.Meth., A620:227-251, 2010.

[14] Pedro Abreu et al. Antennas for the Detection of Radio Emission Pulses from Cosmic-Ray. JINST, 7:P10011, September 2012.

[15] D. García-Fernández et al. On timing accuracy in observing radio impulses associated with Extensive Air Showers. In this conference.

[16] R. Dallier et al. Direct measurement of the vertical component of the electric field from EAS. In this conference.

[17] Benoît Revenu et al. Coherent radio emission from the cosmic ray air shower sudden death. In proceedings of the 33rd ICRC, Rio de Janeiro, Brasil, number 398, July 2013.

[18] D. García-Fernández et al. Near-field radio emission induced by extensive air showers. In this conference.

[19] A. Escudie et al. Low frequency observations of cosmic ray air shower radio emission by CODALEMA/EXTASIS. In this conference. 